\title{
Direct versus indirect revascularization in the treatment of moyamoya disease
}

\author{
*Seong-eun Park, BSc, Ju-seong Kim, MD, Eun Kyung Park, MD, Kyu-Won Shim, MD, PhD, and \\ Dong-Seok Kim, MD, PhD
}

Department of Pediatric Neurosurgery, Severance Hospital, Yonsei University, College of Medicine, Seoul, South Korea

\begin{abstract}
OBJECTIVE For patients with moyamoya disease (MMD), surgical intervention is usually required because of progressive occlusion of the internal carotid artery. The indirect bypass method has been widely accepted as the treatment of choice in pediatric patients. However, in adult patients with MMD, the most effective treatment method remains a matter of debate. Here, the authors compared the clinical outcomes from MMD patients treated with either extracranial-intracranial arterial bypass (EIAB; 43 hemispheres) or modified encephaloduroarteriosynangiosis (mEDAS; 75 hemispheres) to investigate whether mEDAS is an effective surgical method for treating adults with symptomatic MMD.
\end{abstract}

METHODS A comparative analysis was performed in patients treated using either mEDAS or EIAB. Collateral grading, collateral vein counting, and symptom analysis were used to assess the outcome of surgery.

RESULTS Seventy-seven percent (58/75) of mEDAS cases and 83.7\% (36/43) of EIAB cases in the analysis experienced improvement in their symptoms after surgery. Furthermore, patients in $98.7 \%(74 / 75)$ of mEDAS cases and those in $95.3 \%(41 / 43)$ of EIAB cases exhibited improved collateral grades. Increases in regions of perfusion were seen after both procedures.

CONCLUSIONS Modified EDAS and EIAB both result in positive outcomes for symptomatic adults with MMD. However, when considering the benefit of both surgeries, the authors propose mEDAS, a simpler and less strenuous surgery with a lower risk of complications, as a sufficient and safe treatment option for symptomatic adults with MMD.

https://thejns.org/doi/abs/10.3171/2017.5.JNS17353

KEY WORDS moyamoya; encephaloduroarteriosynangiosis; EDAS; revascularization; vascular disorders

$\mathrm{M}$ oyamoya disease (MMD), an idiopathic disease first reported by Suzuki and Takaku in $1969,{ }^{21}$ results from a progressive occlusion and stenosis of the internal carotid artery (ICA). Occlusion typically occurs bilaterally and is accompanied by the development of abnormal net-like vessels called moyamoya vessels. The disease seems to be prevalent in the Asian population, where it is common in pediatric patients in the 1st decade of life and in 30- to 40-year-old adults. Indirect encephaloduroarteriosynangiosis has been a widely estab- lished treatment strategy for children with MMD because of its excellent postoperative results, leading to extensive collateral formation and minimal complications. ${ }^{6,15,16}$ In adults, however, the most efficient treatment strategy for ischemic patients is a matter of debate. Direct bypass surgery has gained popularity because it provides an immediate increase in perfusion. . $^{7,8,17,23}$ However, more studies are highlighting the effectiveness of indirect surgery. ${ }^{20}$ Furthermore, authors of many reports are now suggesting that a combination of direct and indirect methods is beneficial

ABBREVIATIONS DSA = digital subtraction angiography; EIAB = extracranial-intracranial arterial bypass; ICA = internal carotid artery; ICU = intensive care unit; LOS = length of stay; $M C A=$ middle cerebral artery; $\mathrm{mEDAS}$ = modified encephaloduroarteriosynangiosis; MMD = moyamoya disease; MRA = magnetic resonance angiography; STA $=$ superficial temporal artery; TIA $=$ transient ischemic attack.

SUBMITTED February 13, 2017. ACCEPTED May 8, 2017.

INCLUDE WHEN CITING Published online October 27, 2017; DOI: 10.3171/2017.5.JNS17353.

* Ms. S. E. Park and Dr. J. S. Kim contributed equally to this work. 
for patients, as this can establish immediate blood flow from direct surgery and can lead to a prolonged increase in perfusion via an indirect effect. ${ }^{1,3,11,22}$

Initially, because of the popularity of direct bypass surgery, surgeons at our institution mainly performed extracranial-intracranial arterial bypass (EIAB) for treating adults with MMD. However, on long-term follow-up digital subtraction angiography (DSA), instead of improvement in local perfusion resulting from direct bypass, an indirect effect of formation of new vessels in the hypoperfused regions of the brain seemed to be prevalent (Fig. 1). Considering that EIAB is a much more complicated surgery and has been reported to carry a relatively high risk of postoperative hyperperfusion that can lead to irreversible neurological damage, ${ }^{5,12}$ surgeons at our institution began performing indirect modified encephaloduroarteriosynangiosis (mEDAS) surgery. Since there was no consensual agreement on which surgical method was superior in the treatment of adult MMD at the time, ${ }^{2,20}$ a comparative analysis assessing the efficacy of the surgeries seemed necessary. In this study, we present and discuss the clinical outcomes of symptomatic adult MMD patients who underwent either mEDAS or EIAB surgery at our institution, to determine whether mEDAS is a sufficient and safe method for treating adult MMD patients.

\section{Methods \\ Patient Selection}

With the idea that mEDAS surgery on its own may be more beneficial than EIAB for the treatment of adult MMD patients, we compared patients (age $\geq 20$ years at the time of surgery) who were treated for MMD with either indirect bypass mEDAS (between January 2005 and December 2014) or direct EIAB surgery (between October 2011 and May 2015). Between 2005 and 2011, both direct and indirect bypass surgeries were performed. Direct bypass surgeries were favored more often, but in cases in which direct bypass was not feasible (for example, when there were no suitable vessels for the bypass graft) indirect mEDAS was performed. However, when the outcomes of the 2 surgeries were primarily followed up, no significant differences were seen, which led to the simpler mEDAS recently being more favored. All patients underwent surgical treatment at Severance Hospital in Korea. Patients undergoing direct surgery (EIAB) were treated mainly by vascular surgeons, and those undergoing indirect surgery (mEDAS) were treated mainly by pediatric neurosurgeons. Unfortunately, EIAB data prior to 2011 are rather scarce, and the available data are not suitable for a comparative study due to limited materials for analysis. However, as there were detailed data for mEDAS patients between 2005 and 2014, EIAB data from a similar period (2011 and 2015) were used so that a meaningful comparison could be made.

All patients presented with ischemic symptoms including impairment in motor, sensory, cognition, and/or vision. Symptomatic hemispheres were considered for the procedure if they displayed decreased perfusion and/or a lack of vascularity when analyzed on 6-vessel DSA, MRI, and/ or Diamox SPECT. In most patients, the surgical side of the brain hemisphere was in Suzuki Stage II-IV. ${ }^{21}$ Some asymptomatic hemispheres were treated for preventative purposes if they displayed a lack of vascularity (relatively defected capillary filling at angiography or severely delayed time to peak) or if patients complained of severe headache or dizziness (as these are non-hemisphere-specific symptoms, it was deemed asymptomatic) even in the absence of transient ischemic attack (TIA) or infarct. This study was approved by the institutional review board.

\section{Surgical Technique \\ Modified EDAS}

Modified EDAS was performed using a split dura mater as previously described, ${ }^{10}$ with some modifications. After exposure of the superficial temporal artery (STA), the galea was cut parallel to the STA at a distance of $5 \mathrm{~mm}$, so that a strip of galea was attached to the artery over its entire exposed length. The galeal flap was made as long as possible. Then, the temporalis muscle and the pericranium were incised linearly and denuded from the skull. A wider craniotomy than that done for standard EDAS was performed (average diameter $66 \mathrm{~mm}$ ). The linear single dural incision on the outer layer of the dura was made parallel to the galeal flap and the meningeal arteries. The meningeal dura was stripped from the periosteal dura. The meningeal dura was then removed to allow direct contact between the middle meningeal artery and the cerebral cortex. Finally, the remaining periosteal dura was sutured with the galeal flap. Bleeding from the dural incision or separation was controlled using Surgicel (Ethicon) and gentle compression, with minimal use of bipolar coagulation. The elevated bone flap was repositioned to its original site with absorbable material. This procedure developed from the idea of obtaining donor vessels from the dural arterial supply as well as the posterior branch of the STA, thereby being able to insert 2 vessels during a single surgery. The intraoperative $\mathrm{PaCO}_{2}$ levels were strictly controlled between 35 and $45 \mathrm{~mm} \mathrm{Hg}$, and the mean arterial blood pressure was maintained between 100 and $120 \mathrm{~mm} \mathrm{Hg}$. To control postoperative pain from the surgical wounds, an intermittent injection of fentanyl or a continuous drip of morphine $(0.1 \mathrm{mg} / \mathrm{kg}$ in $1 \mathrm{~L}$ of normal saline and $0.035 \mu \mathrm{g} / \mathrm{min} / \mathrm{kg}$, respectively) was used. Postoperative complications were minimized as previously described. ${ }^{13}$

\section{EIAB}

A conventional STA-middle cerebral artery (MCA) anastomosis was carried out. ${ }^{19}$ In brief, the patient was placed supine, and an incision was made directly over the STA branch. The exposed STA was isolated and was mobilized from the galeal layer over the temporalis muscle to prepare for anastomosis. The temporalis muscle was divided and dissected from the underlying bone and then retracted laterally. The dura was opened, and a suitable recipient MCA branch was identified and dissected from the artery.

Temporary clips were placed across the MCA recipient branch to interrupt blood flow. The donor STA was divided at an angle and spatulated for anastomosis. End stitches were placed at the heel and the end of the spatulated artery. An interrupted or running suture array was then placed at the edge of the arteriotomy. After completion of 

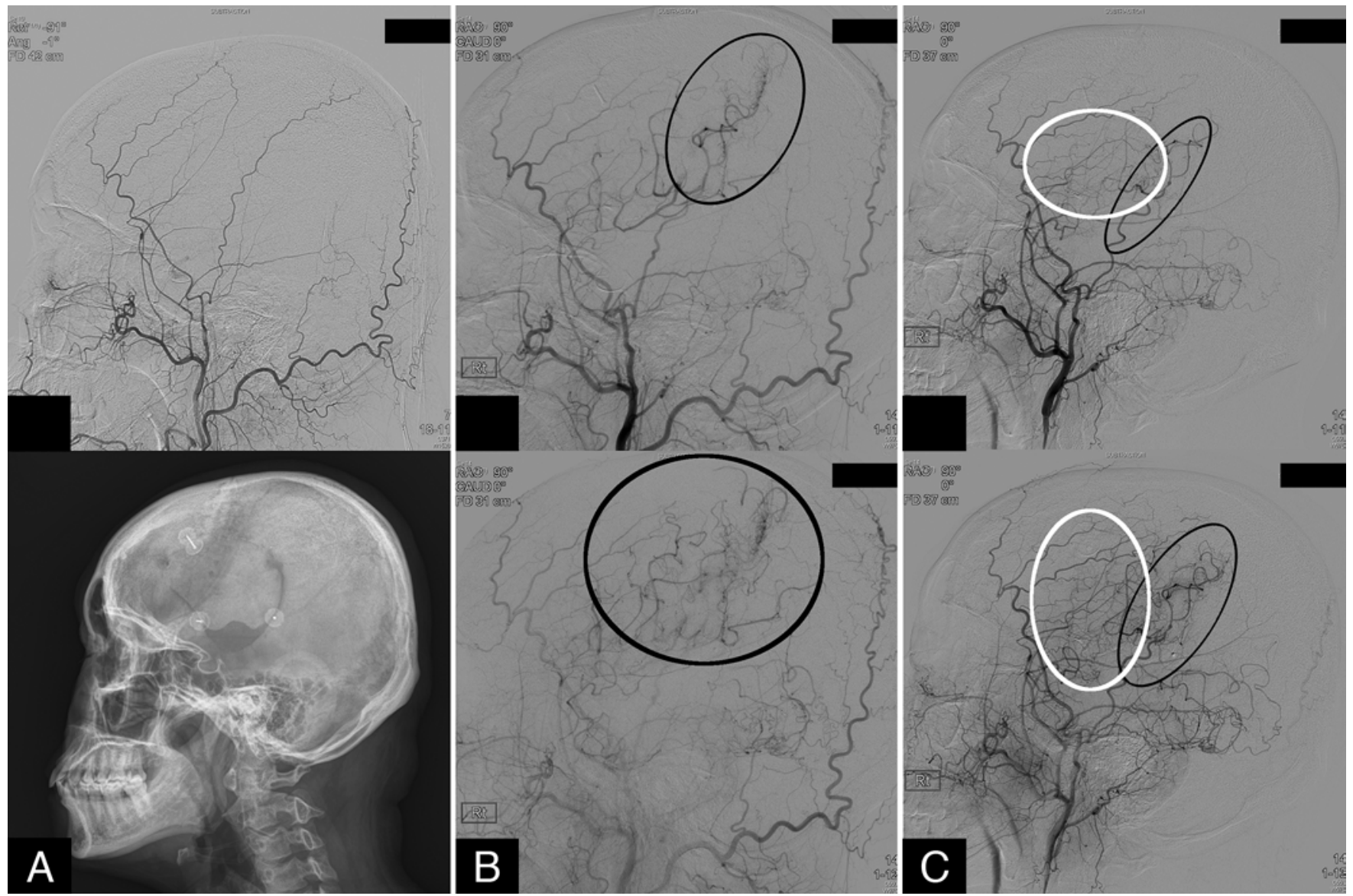

FIG. 1. Indirect effect after direct bypass surgery. This patient underwent EIAB surgery in March 2012. A: Preoperative DS angiogram (lateral view, upper) and image showing the craniotomy site (lower). B: Postoperative DS angiograms (lateral view) obtained in March 2012. Immediate improvement in circulation from the direct bypass is shown within the black circles. C: Follow-up DS angiograms (lateral view) obtained in August 2014. An indirect effect (white circle), occurring adjacent to the direct bypass site, seems to be prevalent.

the arteriotomy, temporary clamps were removed for flow establishment. Leakage was mitigated with Surgicel.

\section{Postoperative Follow-Up \\ Clinical Symptoms}

Follow-up of clinical symptoms, based on patient reporting, was done at the outpatient clinic. As all patients had ischemic MMD, mostly motor symptoms were reported with no significant cognitive decline.

\section{Postoperative MMD MRI Protocol}

The routine postoperative follow-up was performed using an MRI protocol that was specially designed at our institution. The development of collateral vasculature and the progression of MMD were examined using magnetic resonance angiography (MRA). Three-dimensional timeof-flight MRA (TR $8.6 \mathrm{msec}$, TE $12 \mathrm{msec}, 7^{\circ}$ flip angle) was performed using a 3.0-T system (InteraAchieva, Philips Medical Systems) with an 8-channel sensitivityencoded head coil. The orientation of the volume slab was chosen to cover the circle of Willis, posterior cerebral artery, and MCA. The slab was $64 \mathrm{~mm}$ thick with 64 partitions, resulting in a section thickness of $1 \mathrm{~mm}$. The acquisition matrix was $512 \times 512$, and the FOV measured $23 \mathrm{~cm}$. Echo planar imaging was performed during an intravenous bolus injection of $0.2 \mathrm{mmol} / \mathrm{kg}$ of gadopentetate dimeglumine (Magnevist, Schering AG) with the following parameters: TR $8.6 \mathrm{msec}$, TE $12 \mathrm{msec}$, number of acquisitions 1 , flip angle $7^{\circ}$, echo planar imaging factor 7 , section thickness $3.5 \mathrm{~mm}$ without a gap, a FOV $23 \mathrm{~cm}$, and an acquisition matrix of $128 \times 128$.

\section{Image Analysis \\ Collateral Grade}

Preoperative and postoperative MRA results were evaluated by a medical team ( 2 radiologists and 2 neurosurgeons) who made diagnoses by consensus. Postoperative MR angiograms were compared with preoperative studies to evaluate the transdural collateral vessels and monitor changes in STA and moyamoya vessels.

Collateral Grades 1,2, and 3 were determined from DS angiograms and/or MR images according to the extent of formation of transdural involvement in the MCA territory at the synangiosis site (adapted from conventional angiography criteria) according to the grading system of Nakashima et al. (Fig. 2). ${ }^{18}$ 


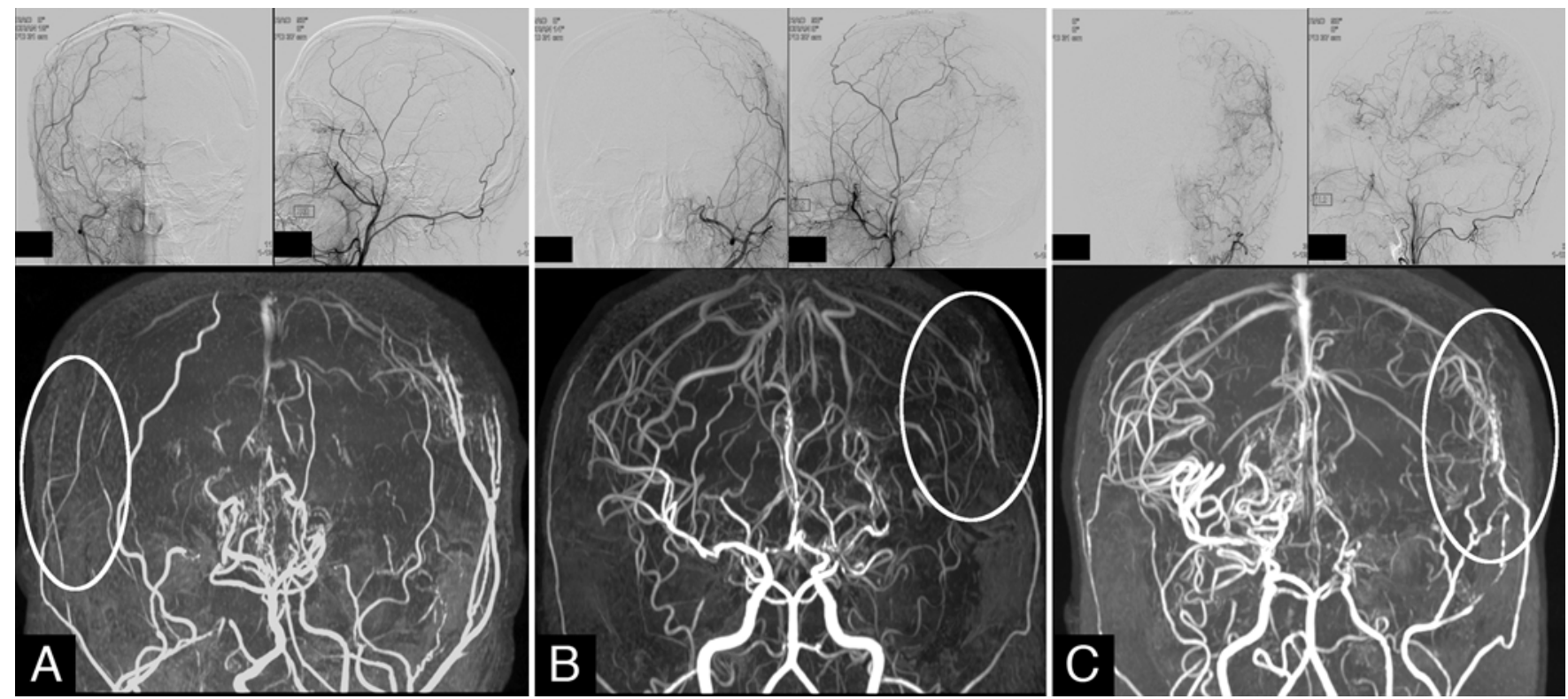

FIG. 2. Collateral grade determination based on DSA (upper [in each panel, anteroposterior and lateral views appear on the left and right, respectively]) and MRA (lower). Regions of interest are marked with white circles on the MRA scans. A: Grade 1. Thickening of STA is seen on both DSA and MRA. B: Grade 2. Focal collateral seen on DSA, where there is initiation of cortical collateral development. On MRA, collaterals are displayed as spots ("spot sign") C: Grade 3. Broad collateral seen on the DS angiogram, where there is network formation between collateral circulation parenchymal vessels. On MRA, the collaterals are connected and appear as a weblike network.

\section{Collateral Vein Count}

For a more quantitative analysis of the extent of collateral formation, using a DS angiogram or 3D-TOF-MR angiogram we counted the number of cortical veins in the ICA system into which the external carotid artery system drains on a DS angiogram or 3D-TOF-MR angiogram (Fig. 3). After craniotomy, collaterals form in the craniotomy site and ischemic regions beyond the craniotomy site. These collaterals drain into several drainage veins in the ICA circulation. The number of drainage veins, therefore, is representative of the extent of collateral formation.

\section{Results}

\section{Patient Demographics}

Modified EDAS surgery was performed in 74 hemispheres in 57 adult MMD patients at our institution (Table 1). Two additional patients who had undergone unilateral EDAS at another hospital underwent contralateral mEDAS surgery at our institution. In these patients, the outcomes of the surgically treated hemispheres at another hospital were included in our data analysis. However, 1 patient who underwent unilateral surgery was lost to follow-up. Therefore, data from 75 surgeries were used in our data analysis.

EIAB was performed in 44 hemispheres in 41 adult MMD patients. However, 1 patient was excluded from the outcome data analysis because follow-up was done at another hospital. Therefore, clinical outcome data from 43 hemispheres were included in our study.

The mean patient age at the time of surgery was 28.7 years for mEDAS and 36.0 years for EIAB. The median follow-up durations for mEDAS and EIAB were 42 months (range 6-132 months) and 22 months (range 1-45 months), respectively. Most patients presented with motor symptoms prior to surgery, and a few patients presented with sensory, cognitive, and visual symptoms. Most surgeries were carried out in hemispheres at Suzuki Stages II-IV.

\section{Measurement of Operative Time}

Operative times were recorded by the anesthetist in the operating room where the initiation and termination of the surgery was clarified by the main surgeon. The mean operative time for EIAB surgery was 397 minutes (250-565 minutes), whereas for mEDAS surgery it was 109 minutes (91-225 minutes).

\section{Length of Postsurgical Stay in the Hospital and the ICU}

Hospital and intensive care unit (ICU) lengths of stay (LOSs) were recorded as indicated in the patient chart. mEDAS patients had an average hospital LOS of 5 days, and no ICU stay after the surgery. EIAB patients had an average hospital LOS of 20 days, and an ICU stay for at least 1 week after the surgery.

\section{Clinical Symptom Improvement}

Follow-up of clinical symptoms was performed in the outpatient clinic. Of the 75 mEDAS surgeries, patients in 51 cases $(68 \%)$ exhibited no significant symptoms, and patients in 7 cases $(9.3 \%)$ presented with decreased frequency of TIAs after surgery. In total, patients in $77.3 \%$ of cases exhibited symptom improvement. Unfortunately, patients in 6 cases (8\%) had an increased frequency of TIAs in the immediate postoperative period, and those in 


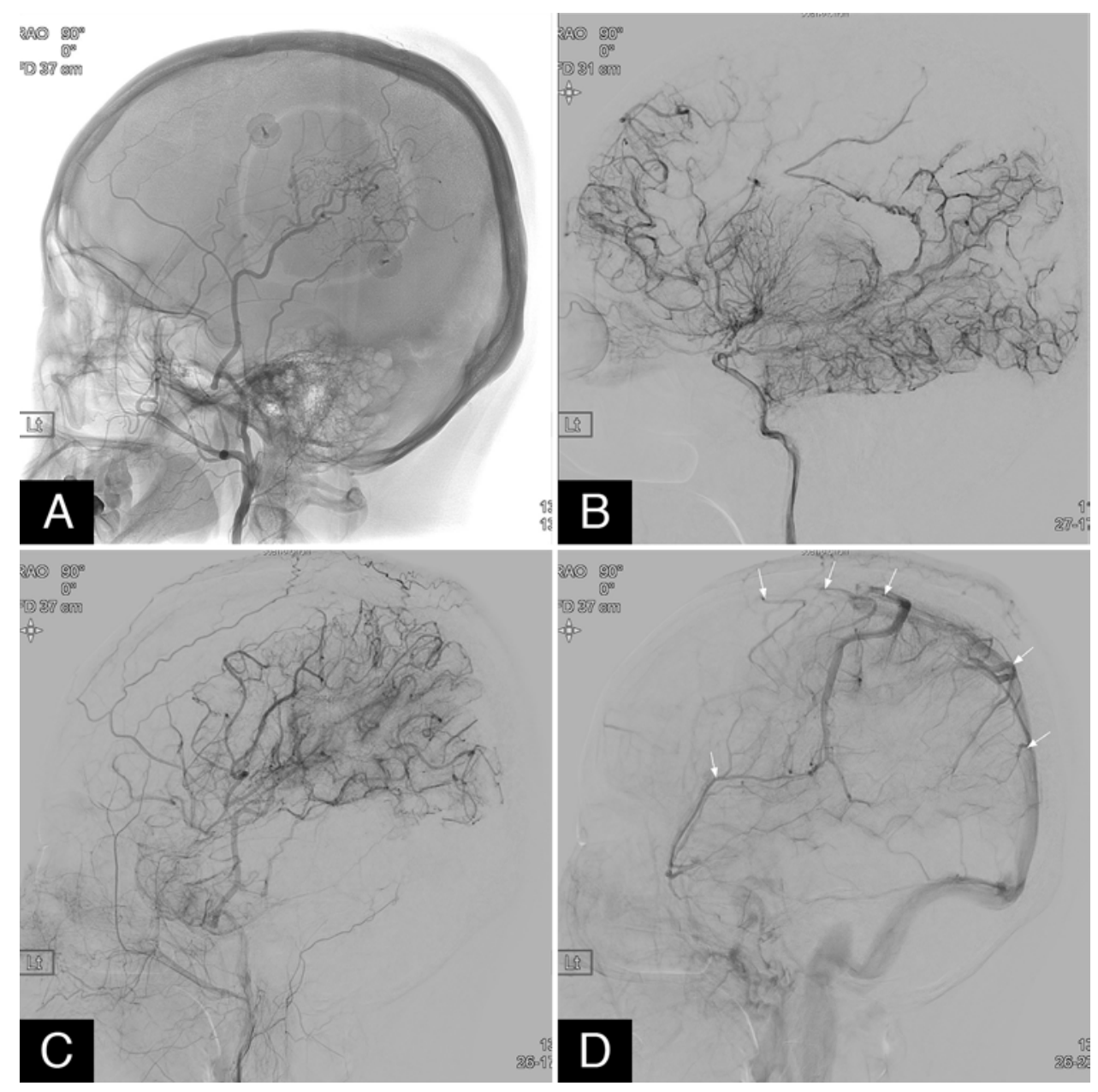

FIG. 3. Cortical veins in the ICA circulation were counted on DS angiograms (lateral views). A: The craniotomy site is shown. B: Regions that lack vessels are shown. The ischemic regions are larger than the craniotomy site. C: The collaterals generated after mEDAS surgery are seen to populate the ischemic regions shown in panel B. D: Veins counted in the ICA circulation (arrows). These were absent on the preoperative DS angiogram but were present on postoperative DS angiogram.

11 cases (14.7\%) suffered cerebral infarction. Of these 11 cases, patients in 7 cases suffered infarction in the preoperative period. This indicates that $63.6 \%$ (7 of 11) of the cases in which patients with infarct complication in the postoperative period are those with a higher risk of infarction from the natural progression of the disease.

Of the 43 EIAB surgeries, patients in 27 cases $(62.8 \%)$ exhibited no symptoms, and those in 9 cases (20.9\%) experienced decreased TIA frequency. In total, patients in $83.7 \%$ of the cases exhibited improved symptoms. However, patients in 5 cases $(11.6 \%$ ) had an increased frequency of TIAs and those in 2 cases $(4.7 \%)$ experienced cerebral infarction . In addition, there were 2 cases of wound dehiscence. These were treated immediately and did not lead to long-term complications.

\section{Collateral Grade}

In addition to symptom analysis, the collateral grades in patients were determined according to the extent of collateral vessel formation after surgery. Here, we considered a collateral grade of 1 or more a positive outcome because patients with Grade 1 collaterals exhibited some level of collateral formation, and the collateral grade progressively improved with time $(\mathrm{p}<0.01)$ for both EIAB and mEDAS surgeries (Fig. 4). On this basis, we can conclude that $98.7 \%$ of mEDAS surgeries led to favorable collateral grades. The collateral vessels were Grades $0,1,2$, and 3 in $1,19,27$, and 28 cases, respectively. For EIAB cases, 95.3\% had favorable collateral grades; the collaterals were Grades $0,1,2$, and 3 in 2, 5, 24, and 12 cases at Grade 3, respectively.

\section{Collateral Drainage Vein}

Although the collateral grade is a good way of measuring the outcomes after surgery, we devised a new method of grading that is more quantitative and objective. To determine the extent of territories populated by collateral vessels, we viewed the DS angiogram or 3D-TOF-MR angiogram to count the number of cortical veins in the ICA circulation into which the regions of collaterals drain. Of the hemispheres treated, 20 mEDAS hemispheres and 35 EIAB hemispheres have undergone postoperative angiographic follow-up, and 53-mEDAS and 8-EIAB hemispheres have undergone 3D-TOF-MRA follow-up. Two 
TABLE 1. Preoperative patient demographics

\begin{tabular}{|c|c|c|}
\hline Variable & mEDAS & EIAB \\
\hline Mean age in yrs (range) & $28.7(20-48)$ & $36.0(19-61)$ \\
\hline \multicolumn{3}{|l|}{ Sex } \\
\hline Female & 42 & 32 \\
\hline Male & 15 & 9 \\
\hline \multicolumn{3}{|l|}{ Bilateral moyamoya surgery* } \\
\hline Yes & 19 & 2 \\
\hline No & 38 & 38 \\
\hline \multicolumn{3}{|l|}{ Preop symptoms $†$} \\
\hline Motor & 40 & 27 \\
\hline Sensory & 3 & 2 \\
\hline Cognitive & 2 & 0 \\
\hline Vision & 2 & 0 \\
\hline \multicolumn{3}{|l|}{ Multiple symptoms } \\
\hline Motor \& sensory & 4 & 6 \\
\hline Motor \& vision & 2 & 3 \\
\hline Motor, sensory, \& cognition & 1 & 0 \\
\hline Motor, sensory, \& vision & 0 & 2 \\
\hline Nonspecific symptoms $\ddagger$ & 3 & 1 \\
\hline \multicolumn{3}{|l|}{ Preop Suzuki stage† } \\
\hline I & 3 & 1 \\
\hline II & 15 & 14 \\
\hline III & 34 & 22 \\
\hline IV & 20 & 7 \\
\hline $\mathrm{V} \S$ & 2 & 0 \\
\hline IV§ & 1 & 0 \\
\hline \multicolumn{3}{|c|}{$\begin{array}{l}\text { * Number of patients. } \\
\dagger \text { Number of hemispheres. } \\
\text { † Headaches and dizziness. } \\
\S \text { Surgery was performed at late Suzuki stages because of severe symptom } \\
\text { presentation. }\end{array}$} \\
\hline
\end{tabular}

mEDAS-treated hemispheres were excluded from vein count as the MRA scans were insufficient for vein counting. A larger number of affected veins represent a larger area of collateral formation, indicating effectiveness of the surgery. As shown in Fig. 5, there is a strong positive correlation $(p<0.01)$ between collateral grades and number of drainage veins, where the number of veins increased as grades increased in both groups.

\section{Discussion}

Adult patients with symptomatic MMD have a chronic decrease in blood flow. It is therefore necessary to establish good collateral circulation to prevent the risks of ischemia and hemorrhage. ${ }^{23}$ To date, it has not been decided whether direct or indirect surgery is best for treating adults with symptomatic MMD. Through this study, we hope to impart our view that mEDAS is a sufficient surgical method for adult MMD treatment and that it is more beneficial for patients than EIAB.

In this study, for choosing the method of intervention, patients and their families decided on the treatment option after receiving sufficient information on both mEDAS and
EIAB procedures. As this was not a process that could be randomized, some differences in the patient demographics were inevitable. Patient age and Suzuki stages at the time of surgery differ slightly between mEDAS and EIAB. For mEDAS patients, the age at operation varied between 20 and 48 years, with $56 \%$ of patients being $20-30$ years at the time of surgery. On the other hand, for EIAB patients, the age at operation ranged from 19 to 61 years, and $44 \%$ of patients were between 30 and 40 years old at the time of surgery. Considering Suzuki stages, $16 \%$ of EIAB cases were higher than Stage III, whereas $31 \%$ of mEDAS cases were higher than Stage III. Therefore, in general, mEDAS patients were younger but had more advanced MMD, and EIAB patients were slightly older but had less advanced MMD. As mEDAS has proven to be effective in pediatric patients, it is expected that the surgery will yield beneficial results when performed in younger adults. For advancedstage MMD, if the patient is older, EIAB surgery carries higher risks as it destroys natural collaterals. Therefore, given that the outcome of mEDAS and EIAB surgeries are similar, mEDAS could be used not only in younger adult patients, but also in older patients with advanced MMD as an alternative method to EIAB.

Most studies of adult MMD focus on patients with hemorrhagic MMD. At our institution, however, patients with hemorrhagic MMD are not candidates for mEDAS. Although it has been indicated that direct and/or indirect bypass surgery could reduce the risk of recurrent hemorrhage in hemorrhagic patients, ${ }^{17}$ the results are limited. ${ }^{4}$ Due to the lack of definitive evidence supporting the positive effect of bypass surgery on patients with hemorrhagic MMD, these patients usually receive supportive management, such as catheter insertion for hematoma removal or relief of hemorrhagic hydrocephalus.

The mean follow-up periods were 42 months (range 6-132 months) and 22 months (range 1-45 months) for mEDAS and EIAB patients, respectively. The difference in the follow-up duration is due to the fact that data from EIAB surgeries were more recent than those from mEDAS procedures. However, despite the difference, the follow-up duration for EIAB was comparable to that in other MMD studies (32.7 months in the study by Gazyakan et al., and 6 months to 2 years in the study by Li et al. ${ }^{14}$ ) and, with the presence of reports showing that that there are no significant changes in clinical outcome 3 years after surgery, ${ }^{17}$ we deemed that it was a representative length of follow-up for assessing the outcome.

\section{Assessment of Clinical Outcomes and Complications}

In recording the clinical presentations of the patients in our study, we found that $77.3 \%$ of mEDAS surgeries and $83.7 \%$ of EIAB surgeries led to improved clinical symptoms of MMD. Although the percentage of patients with improved clinical symptoms was higher after EIAB, it was relatively clear that mEDAS surgery also led to good outcomes, which is why the mEDAS surgery was not abandoned.

Unfortunately, TIA and infarction incidents occurred after mEDAS (8\% and $14.7 \%$, respectively) and EIAB $(11.6 \%$ and $4.7 \%$, respectively). Because the patients in our study had symptomatic MMD, the presence of TIA 


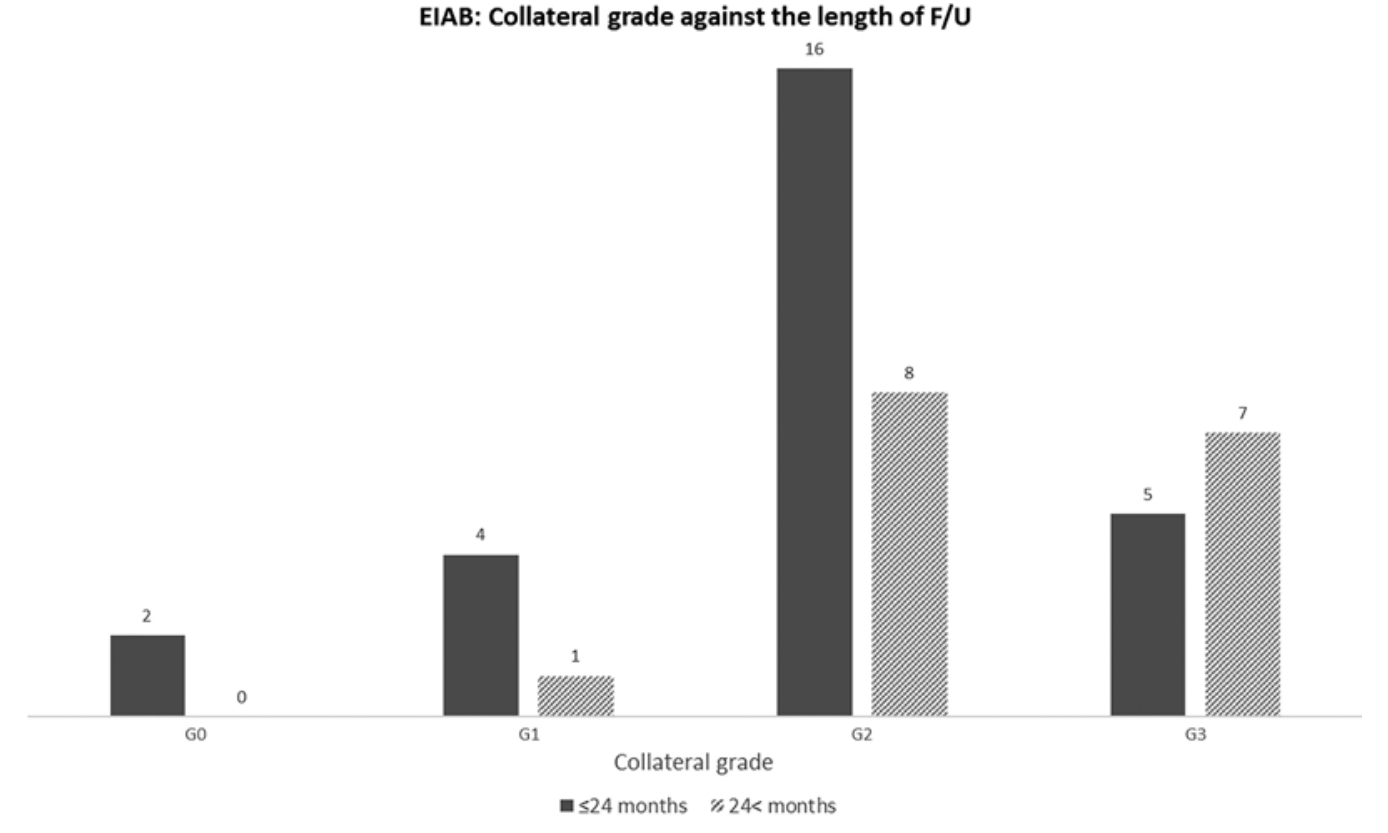

mEDAS: Collateral grade against the length of $F / U$

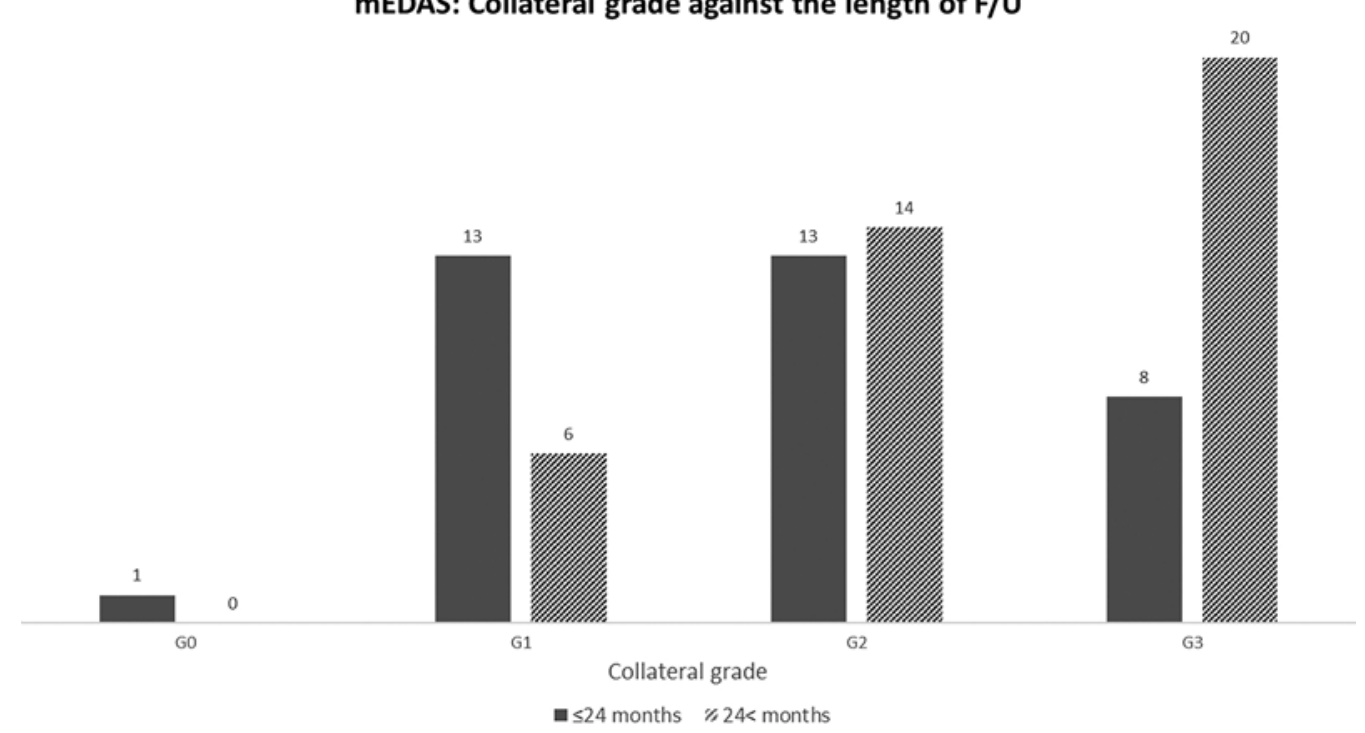

FIG. 4. Change in collateral grading against length of follow-up for both EIAB and mEDAS patients. Upper: Collateral grades for the 43 EIAB surgeries. The number of cases at Grade 3 increased with time, whereas those for Grades 0 , 1 , and 2 all decreased. The effect was not as prevalent, as only 16 patients were underwent follow-up for more than 24 months, whereas 27 patients underwent follow-up for less than 24 months. Lower: Collateral grades for the 75 mEDAS surgeries. Cases at Grades 2 and 3 increased, while cases at Grade 1 decreased with time, indicating that the collateral grade increases with the length of follow-up $(F / U)(p<0.01)$.

and infarction may stem from the natural progression of the disease, rather than from surgical complication. It has been previously indicated that the occurrence of preoperative infarction is associated with a higher risk of postoperative complications. ${ }^{9,13}$ As $63.6 \%$ of the postoperative infarct patients in the mEDAS group suffered infarction in the preoperative period, this factor may contribute to the relatively high level of infarction in mEDAS patients. Infarct patients had unstable blood perfusion and advanced disease stage, suggesting that they generally did not have suitable vessels for direct bypass surgery. In these cases, the simpler mEDAS was preferred over EIAB, which contributes to the skewing of preoperative infarction occurring in patients with advanced stage disease to mEDAS. Even so, an additional method for controlling complications, beyond what is already in place, should be developed to further decrease perioperative ischemic complications.

The results of our study show that $98.6 \%$ of mEDAS cases and $95.3 \%$ of EIAB cases had positive postoperative collateral grades where we could see postoperative collateral formation. Furthermore, for both EIAB and mEDAS cases, the proportion of cases at Grade 2 or 3 were higher 


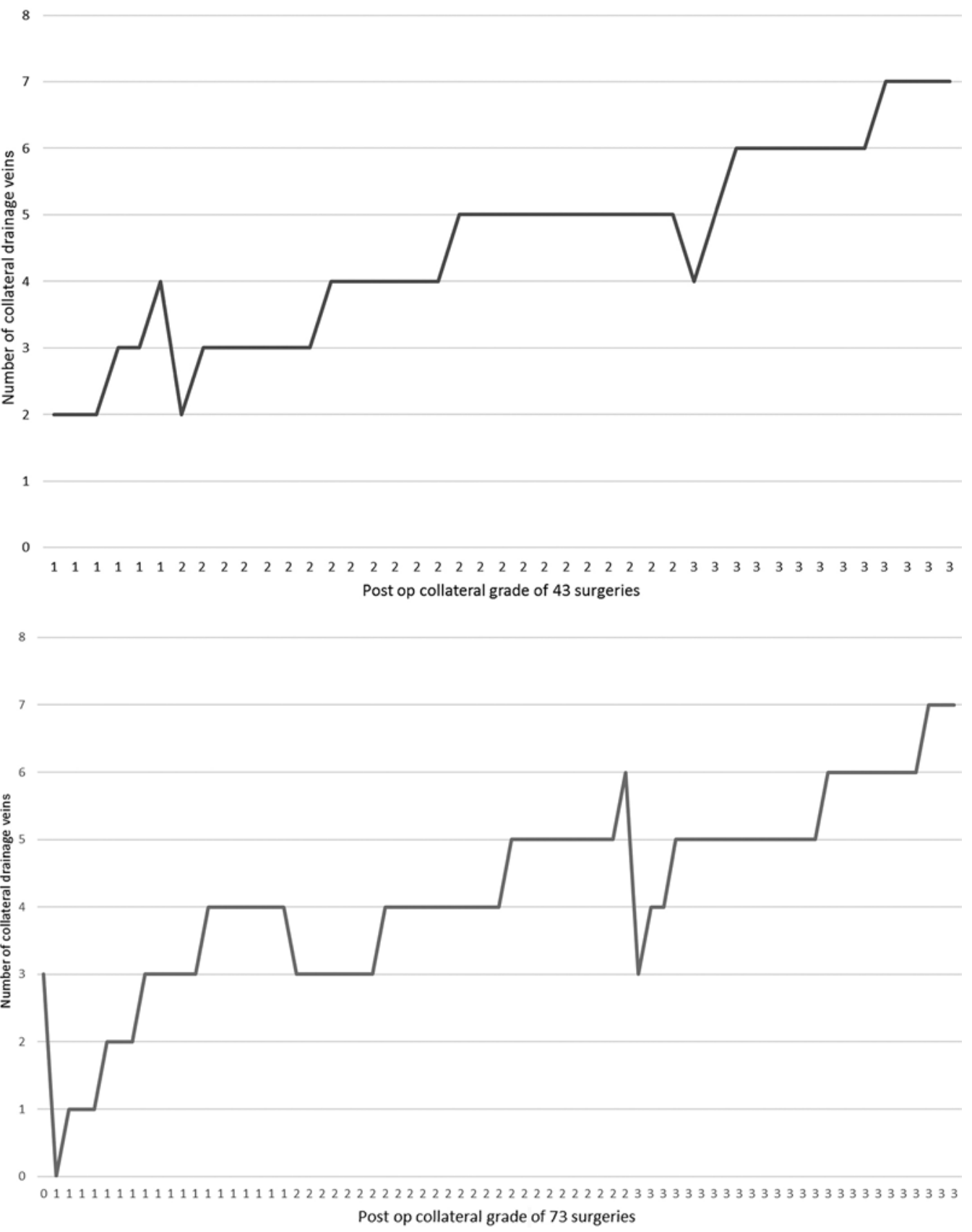

FIG. 5. Graphs showing the number of collateral drainage veins at each collateral grade. Strong positive correlation between postoperative collateral grades and the number of collateral drainage veins are seen in both cases $(p<0.01)$. Upper: For EIAB surgeries, there were 2-4 Grade 1 veins, 2-5 Grade 2 veins, and 4-7 Grade 3 veins. Lower: For mEDAS surgeries, there were 3 Grade 0 veins, $0-4$ Grade 1 veins, 3-6 Grade 2 veins, and 3-7 Grade 3 veins.

when the patients underwent follow-up for more than 24 months rather than less than 24 months $(77.8 \%$ to $93.8 \%$ for EIAB patients and $60 \%$ to $85 \%$ for mEDAS patients). This indicates a general trend of collateral grade improvement upon increase in follow-up length. Therefore, even if the collateral grade at the time of follow-up is not as high as expected, it is likely that it will improve with time. Although this effect is seen in both groups, the effect for EIAB patients may be due to an indirect effect from craniotomy, which facilitates improvement of grade over time.
This is an important factor to consider when interpreting results from direct bypass surgeries, because these results not only reflect the direct effect of the surgery, but also include the indirect bypass effect generated from craniotomy.

In this study, we devised a more quantitative and objective method of determining the extent of collateral formation, which involves counting the drainage veins in the ICA circulation. We found a strong positive correlation between collateral grades and collateral drainage veins 
in both surgical groups (Fig. 5). As collateral grade was shown to improve with time, we also expect the number of collateral drainage veins to increase with time. This indicates that there will be a progressive increase in the area perfused by collateral vessel formation, which fulfills the objective of the surgery and also yields comparable outcome to EIAB surgery.

The modified Rankin Scale score is commonly used for assessing the outcome of patients; however, we did not use the modified Rankin Scale, as all patients were at Score 0 or 1, and no significant data set could be generated.

\section{Patient Experience}

The results that we have presented, based on symptom analysis, collateral grading, and collateral vein count, suggest that the outcomes generated by mEDAS and EIAB surgeries were not significantly different. However, when we considered patient experience, a clear difference became obvious. The mean operative time for EIAB surgery was 397 minutes, 3.5 times longer than the 109 minutes for mEDAS surgery, indicating that EIAB patients went through a much more strenuous surgical process. Also, the hospital and ICU LOSs were shorter for mEDAS patients than for for EIAB patients. Patients who underwent mEDAS had an average hospital LOS of 5 days and did not have to stay in the ICU after surgery. In contrast, EIAB patients had a hospital LOS of approximately 20 days, and they had to stay in the ICU for at least 1 week after surgery. This significant difference in overall ICU and hospital LOSs suggests that mEDAS is a simpler surgery that does not disrupt the patient's life as much as EIAB surgery. Further supporting our arguments for choosing mEDAS are studies showing that some EIAB patients have reported complications of hyperperfusion syndrome after surgery. ${ }^{5,12}$ Because MMD patients present with ischemic symptoms due to unstable perfusion, hyperperfusion syndrome is a serious complication that needs to be taken into consideration.

\section{Conclusions}

It would be difficult to argue against the efficacy of EIAB surgery for the treatment of adults with symptomatic MMD. This method has been proven to establish immediate blood flow in regions with decreased perfusion, and multiple reports have presented favorable outcomes stemming from EIAB surgeries. Our goal in this study, however, was not to dispute the efficiency of EIAB as a treatment option, but to consider which surgical intervention provides greater benefits for MMD patients. The clinical outcome should be considered as one of the most important factors for choosing the surgery. However, the risks of surgery and the quality of patient experience should also be considered. Modified EDAS, when compared with EIAB, is a surgery that ensures shorter operative times, shorter ICU and hospital LOSs, and decreased risks of complication. Also, mEDAS has been shown to produce positive clinical outcomes, which, over time, will be comparable to those from EIAB. In addition, we have shown that mEDAS patients do not suffer from serious complications, whereas EIAB patients run the risk of hyperperfusion. Therefore, considering both the clinical outcomes and patient experi- ence aspect of the 2 treatments, we believe that mEDAS is at the least an equivalently effective treatment option for adult symptomatic MMD patients as EIAB.

\section{References}

1. Amin-Hanjani S, Singh A, Rifai H, Thulborn KR, Alaraj A, Aletich V, et al: Combined direct and indirect bypass for moyamoya: quantitative assessment of direct bypass flow over time. Neurosurgery 73:962-968, 2013

2. Baaj AA, Agazzi S, Sayed ZA, Toledo M, Spetzler RF, van Loveren H: Surgical management of moyamoya disease: a review. Neurosurg Focus 26(4):E7, 2009

3. Cho WS, Kim JE, Kim CH, Ban SP, Kang HS, Son YJ, et al: Long-term outcomes after combined revascularization surgery in adult moyamoya disease. Stroke 45:3025-3031, 2014

4. Derdeyn CP: Direct bypass reduces the risk of recurrent hemorrhage in moyamoya syndrome, but effect on functional outcome is less certain. Stroke 45:1245-1246, 2014

5. Fujimura M, Kaneta T, Mugikura S, Shimizu H, Tominaga T: Temporary neurologic deterioration due to cerebral hyperperfusion after superficial temporal artery-middle cerebral artery anastomosis in patients with adult-onset moyamoya disease. Surg Neurol 67:273-282, 2007

6. Fujita K, Tamaki N, Matsumoto S: Surgical treatment of moyamoya disease in children: which is more effective procedure, EDAS or EMS? Childs Nerv Syst 2:134-138, 1986

7. Gazyakan E, Lee CY, Wu CT, Tsao CK, Craft R, Henry SL, et al: Indications and outcomes of prophylactic and therapeutic extracranial-to-intracranial arterial bypass for cerebral revascularization. Plast Reconstr Surg Glob Open 3:e372, 2015

8. Guzman R, Lee M, Achrol A, Bell-Stephens T, Kelly M, Do $\mathrm{HM}$, et al: Clinical outcome after 450 revascularization procedures for moyamoya disease. Clinical article. J Neurosurg 111:927-935, 2009

9. Hyun SJ, Kim JS, Hong SC: Prognostic factors associated with perioperative ischemic complications in adult-onset moyamoya disease. Acta Neurochir (Wien) 152:1181-1188, 2010

10. Kashiwagi S, Kato S, Yamashita K, Takasago T, Akimura T, Okamura S, et al: Revascularization with split duro-encephalo-synangiosis in the pediatric moyamoya disease-surgical result and clinical outcome. Clin Neurol Neurosurg 99 (Suppl 2):S115-S117, 1997

11. Kim DS, Huh PW, Kim HS, Kim IS, Choi S, Mok JH, et al: Surgical treatment of moyamoya disease in adults: combined direct and indirect vs. indirect bypass surgery. Neurol Med Chir (Tokyo) 52:333-338, 2012

12. Kim JE, Oh CW, Kwon OK, Park SQ, Kim SE, Kim YK: Transient hyperperfusion after superficial temporal artery/ middle cerebral artery bypass surgery as a possible cause of postoperative transient neurological deterioration. Cerebrovasc Dis 25:580-586, 2008

13. Kim SH, Choi JU, Yang KH, Kim TG, Kim DS: Risk factors for postoperative ischemic complications in patients with moyamoya disease. J Neurosurg 103 (5 Suppl):433-438, 2005

14. Li Z, Zhou P, Xiong Z, Ma Z, Wang S, Bian H, et al: Perfusion-weighted magnetic resonance imaging used in assessing hemodynamics following superficial temporal artery-middle cerebral artery bypass in patients with moyamoya disease. Cerebrovasc Dis 35:455-460, 2013

15. Manceau E, Giroud M, Dumas R: Moyamoya disease in children. A review of the clinical and radiological features and current treatment. Childs Nerv Syst 13:595-600, 1997

16. Matsushima T, Fujiwara S, Nagata S, Fujii K, Fukui M, Kitamura K, et al: Surgical treatment for paediatric patients with moyamoya disease by indirect revascularization procedures 
(EDAS, EMS, EMAS). Acta Neurochir (Wien) 98:135-140, 1989

17. Miyamoto S, Yoshimoto T, Hashimoto N, Okada Y, Tsuji I, Tominaga $\mathrm{T}$, et al: Effects of extracranial-intracranial bypass for patients with hemorrhagic moyamoya disease: results of the Japan Adult Moyamoya Trial. Stroke 45:1415-1421, 2014

18. Nakashima H, Meguro T, Kawada S, Hirotsune N, Ohmoto T: Long-term results of surgically treated moyamoya disease. Clin Neurol Neurosurg 99 (Suppl 2):S156-S161, 1997

19. Newell DW: Superficial temporal artery to middle cerebral artery bypass. Skull Base 15:133-141, 2005

20. Starke RM, Komotar RJ, Connolly ES: Optimal surgical treatment for moyamoya disease in adults: direct versus indirect bypass. Neurosurg Focus 26(4):E8, 2009

21. Suzuki J, Takaku A: Cerebrovascular "moyamoya" disease. Disease showing abnormal net-like vessels in base of brain. Arch Neurol 20:288-299, 1969

22. Xu B, Song DL, Mao Y, Gu YX, Xu H, Liao YJ, et al: Superficial temporal artery-middle cerebral artery bypass combined with encephalo-duro-myo-synangiosis in treating moyamoya disease: surgical techniques, indications and midterm follow-up results. Chin Med J (Engl) 125:4398-4405, 2012
23. Zipfel GJ, Fox DJ Jr, Rivet DJ: Moyamoya disease in adults: the role of cerebral revascularization. Skull Base 15:27-41, 2005

\section{Disclosures}

The authors report no conflict of interest concerning the materials or methods used in this study or the findings specified in this paper.

\section{Author Contributions}

Conception and design: Shim, DS Kim. Acquisition of data: Shim, JS Kim, EK Park, DS Kim. Analysis and interpretation of data: SE Park, JS Kim. Drafting the article: SE Park, JS Kim. Critically revising the article: Shim. Reviewed submitted version of manuscript: all authors. Approved the final version of the manuscript on behalf of all authors: DS Kim. Study supervision: DS Kim.

\section{Correspondence}

Kyu-Won Shim, 50 Yonseiro, Seodaemungu, Seoul, South Korea. email: shimkyuwon@yuhs.ac. 